\title{
Effect of Hydrokinesia in Elderly Subjects with Backward Disequilibrium: A Systematic Review
}

\author{
Radhika Chintamani* , Amrutkuvar Rayjade and Trupti Yadav \\ Faculty of Physiotherapy, Department of Musculoskeletal Sciences, \\ Krishna Institute of Medical Sciences Deemed to be University, Malkapur, Karad - 415539, \\ Maharshatra, India; radds2009@gmail.com
}

\begin{abstract}
Background: Water based exercise are significant in improving both static as well as dynamic balance. Elderly subjects already are known to have decreased balance. Changes in the musculoskeletal system are one of the major causes of decreased balance. Physiotherapy regimen is important in improving both static and dynamic balance in elderly. Aquatic therapy is painless and easy way to improve balance in geriatric. However, despite its widespread knowledge of importance of aquatic therapy in improvement of balance, the efficacy of aquatic therapy in elderly is limited. Purpose: The aim of this systematic review is to analyze the importance of aquatic therapy on fall perspective in elderly population. Methods: The authors searched Google Scholar and PubMed from 1996 to 2017. Only downloadable randomized controlled trials (15), randomized clinical trials (14) and experimental studies (3) were included in the study. Results: The duration of treatment varied significantly in comparison to various studies. Subgroup analysis was performed on factors considered for measuring of fall risk parameters in elderly subjects. Conclusion: The result of the present study shows strong evidence to support the water based exercises in which Hydrokineitic therapy showed significant reduction in kinesiophobia and fall risk rate. Hence the therapy can be considered effective for geriatric population.
\end{abstract}

Keywords: Backward Disequilibrium, Elderly, Systematic Review, Water Based Exercises

\section{Introduction}

Falls are one of the major risks in geriatric population causing mortality and morbidity. People aged 65 years and older fall each year and this ratio increases with age. The cause of fall can be various like; history of previous fall, disturbed gait due to neurological and balance problems, poor vision and chronic diseases such as arthritis ${ }^{1}$.

Backward Disequilibrium (BD) is a postural disorder observed in elderly subjects who have distortion in their perception of vertical posture. It is commonly seen as the age increases, as the distortion in their perception of vertical posture is reduced. $\mathrm{BD}$ commonly may be due to musculoskeletal changes as well as psychological changes. BD may lead to fall of the individual which has many consequences as mentioned above $^{2}$.

Reduction of fall has become one of the major focuses on research nowadays. Some of those interventions are; land based exercises, home safety ergonomic advices, multifactorial interventions and others have shown to be effective. Unfortunately, aquatic therapy is understudied with perspective of fall risk in geriatric population, thus limiting the ability to use traditional systematic review to examine the comparative effectiveness of all published interventions of water based exercise therapy in geriatric population. Hence this study is been undertaken ${ }^{3}$.

\section{Methods}

\subsection{Ethical Clearance}

Ethical clearance was obtained from Institutional ethical committee KIMS Deemed to be University Karad, Maharashtra.

\subsection{Search Strategy}

The author underwent searches of Google scholar, PubMed, Embase (Excerpta Medica Database), Cochrane Library, Web of Science and CINAHL (Cumulative Index to Nursing and Allied Health Literature) with English database being strictly followed in the study.

\subsection{Study Selection}

Reviewer independently selected the articles and downloaded the articles from Google scholar and PubMed. Inclusion criteria: RCTs, Randomized Clinical Trials and Experimental studies

${ }^{*}$ Author for correspondence 
on Aquatic therapy in geriatric subjects with perspective of fall, age greater than 60 years. At least one fall related outcome measure, both genders, all forms of Hydrokinetic exercises, studies or articles which can be downloaded. Exclusion Criteria: Studies with known neurological disorders like stroke, head injury, spinal cord injury, significant musculoskeletal disorders, Passive physiotherapeutic regimen, discontinued physiotherapeutic regimen, Chronic illness like osteoarthritis of greater than grade 3 of Kellegren Lawrence Classification, cardiovascular diseases etc and subjects who were unable to walk due to chronic pain anywhere in body ${ }^{4}$.

\subsection{Quality Assessment}

Quality of the studies recruited in this study was assessed by quality list from Cochrane Collaboration recommendations. Data were extracted independently and checked for accuracy for the purpose of methodological quality.

\subsection{Data Analysis}

Information was extracted from each included trial on: 1. Descriptive characteristics of study (first author, Journal name, published year, Title, sample size); 2. Sample characteristics (average age, >65); 3. Type of intervention; 4. Fall-related outcome measure (length of follow-up, effect of the intervention).

\subsection{Data Extraction}

Following data was extracted from the study: 1. Trial design (sample size and inclusion and exclusion criteria); demographic data of subjects (age, gender, risk of falls); type of intervention (experimental and control components, duration); and outcomes measured as (rate of fall, risk of fall, kinesiophobia, health related quality of life, risk of fracture).

\subsection{Data Synthesis and Statistical Methods}

Statistical heterogeneity of treatment effects between trials was assessed with a significance level at $\mathrm{p}<0.05$, and the $\mathrm{I}^{2}$ statistic, as per the Cochrane handbook guidance.

Table 1. The detailed elaboration of the studies recruited in this systematic review. Given at the last page.

\section{Results}

Electronic searches identified 100 records, after removal of duplicates, screening the titles and abstracts of 75 records was done. Full-text articles were assessed for 65 records and resulted in 32 eligible studies were taken into consideration. 18 RCTs studies involved a total of 693 subjects, among which 363 were in water based exercises group and 330 were in control group/non-water based exercise group. 12 Randomized Clinical Trials consisted of 459 subjects among whom 233 subjects were given with water therapy and 226 various other therapies. And 2 experimental studies in which; 53 sample size was considered.

\section{Conclusion}

Hydrotherapy uses S.W.E.A.T method, Aquatic aerobics and Deep water running form of water based exercises, showed significant effect in reducing the rate of fall in elderly subjects. S.W.E.A.T method principle are; change in surface area and speed, working positions like rebound, neutral, suspended and extended, enlargement of movement, working around the body, travelling through water forward, backward or diagonal. The results indicate a mean difference of 12.59 (95\% CI 0.10 to 40.5$)$.

\section{Funding}

Self funding.

\section{Conflicts of Interest}

No conflicts of interest.

\section{References}

1. Bolding D, Corman E. Falls in the geriatric patient. Clinics in Geriatric Medicine. 2019; 5(1):115-26. PMid: 30390977. https:// doi.org/10.1016/j.cger.2018.08.010

2. Manckoundia P, Mourey F, Perennou D, Pfitzenmeyer P. Clinical interventions in aging. 2008; 3(4):667-72. PMid: 19281059 PMCid: PMC2682399. https://doi.org/10.2147/CIA.S3811

3. Hopewell S, Copsey B, Nicolson P, Adedire B, Boniface G, Lamb S. Multifactorial interventions for preventing falls in older people living in the community: A systematic review and meta-analysis of 41 trials and almost 20000 participants. British Journal of Sports Medicine. 2019; 0:1-13. PMid: 31434659. https://doi. org/10.1136/bjsports-2019-100732

4. Scheets P, Sahrmann S, Norton B, Stith J, Crowner B. What is backward disequilibrium and how do I treat it? Journal of Neurologic Physical Therapy. 2015; 39(2):119-26. PMid: 25742374. https://doi.org/10.1097/NPT.0000000000000084

5. Takeshima N, Rogers M, Watanabe E, Brechue W, Okada A, Yamada T. et al. Water-based exercise improves health related aspects of fitness in older women. Journal of the American College of Sports Medicine. 2001; 34(3):544-551. PMid: 11880822. https://doi.org/10.1097/00005768-200203000-00024

6. Lord S, Thoma. M, Bindon J, Chan D, Haren. A. The effects of water exercise on physical functioning in older 
people. American Journal on Aging. 2006; 25(1):36 -41. https://doi.org/10.1111/j.1741-6612.2006.00138.x

7. Tsourlou T, Benik A, Dipla K, Zafeiridia A, Kellis S. The effects of a twenty-four-week aquatic training program on muscular strength performance in healthy elderly women. Journal of Strength and Conditioning Research. 2006; 20(4):811-8. PMid: 17194242. https://doi.org/10.1519/00124278-200611000-00014

8. Barela A, Duarte M. Biomechanical characteristics of elderly individuals walking on land and in water. Journal of Electromyography and Kinesiology. 2008; 18:446-54. PMid: 17196825. https://doi.org/10.1016/j.jelekin.2006.10.008

9. Candeloro JM, Coromano FA. Effects of a hydrotherapy program on flexibility and muscular strength in elderly women. Rev Bras Fisioter. 2007; 11(4):267-72. https://doi.org/10.1590/S141335552007000400010

10. Melzer I, Elbar O, Tsedek I, Oddsson L. A water-based training program that include Perturbation exercises to improve stepping responses in older adults: Study protocol for a randomized controlled cross-over trial. BMC Geriatric. 2008; 8:19. PMid: 18706103 PMCid: PMC2532994. https://doi.org/10.1186/14712318-8-19

11. Kastura Y, Yoshikawa T, Ueda S, Usui T, Sotobayashi D, Nakao H. et al. Effects of aquatic exercise training using water-resistance equipment in elderly. Eur J Appl Physiol. 2010; 108:957-64. PMid: 19960351. https://doi.org/10.1007/s00421-009-1306-0

12. Bocalini D, Serra A, Rica R, Santos L. Repercussions of training and detraining by water based exercise on functional fitness and quality of life: A short-term follow-up in healthy older women. Clinics. 2010; 65(12):1305-9. PMid: 21340219 PMCid: PMC3020341. https://doi.org/10.1590/S1807-59322010001200013

13. Avelar N, Bastone A, Alcantara M, Gomes W. Effectiveness of aquatic and non-aquatic lower limb muscles endurance training in the static and dynamic balance of elderly people. Rev Bras Fisioter, Sao Carlos. 2010; 14(3):229-36. PMid: 20730368. https://doi.org/10.1590/S1413-35552010000300007

14. Bento P, Pereira G, Ugrinowitsch C, Rodacki A. The effects of a water-based exercise program on strength and functionality of older adults. Journal of Aging and Physical Activity. 2012; 20(4):469-70. PMid: 22714953. https://doi.org/10.1123/japa. 20.4.469

15. Elbar O, Tzedek I, Vered E, Shvarth G, Friger M, Melzer I. A water-based training program that includes perturbation exercises improves speed of voluntary stepping in older adults: A randomized controlled cross-over trial. Archives of Gerontology and Geriatrics. 2012; 56(1):134-40. PMid: 22951028. https://doi. org/10.1016/j.archger.2012.08.003

16. Sanders M, Takeshima N, Rogers M, Colado J, Borreani S. Impact of the S.W.E.A.T. water-exercise method on activities of daily living for older women. Journal of Sports Science and Medicine. 2013; 12:707-15.

17. Kim S, O'Sullivan D. Effects of aqua aerobic therapy exercise for older adults on muscular strength, agility and balance to prevent falling during gait. J Phy Ther Sci. 2013; 25:923-7. PMid: 24259886 PMCid: PMC3820233. https://doi.org/10.1589/ jpts.25.923
18. Kovach M, Plachy J, Bognar J, Balogh Z, Barthalos I. Effects of pilates and aqua fitness training on older adults' physical functioning and quality of life. Biomedical Human Kinetics. 2013; 5:22-7. https://doi.org/10.2478/bhk-2013-0005

19. Oliveira M, Silva R, Dascal J, Teixeira D. Effect of different types of exercise on postural balance in elderly women: A randomized controlled trial. Archives of Gerontology and Geriatrics. 2014; 59:506-14. PMid: 25239512. https://doi.org/10.1016/j. archger.2014.08.009

20. Khanjari Y, Garooei R. The effect of a period of aquatic therapy exercise on the quality of life and depression in aged males suffering from chronic physical pains. International Letters of Social and Humanistic Sciences. 2015; 56:127-37. https://doi. org/10.18052/www.scipress.com/ILSHS.56.127

21. Alberti D, Lazarotto L, Bento P. Effects of a deep-water running program on muscle function and functionality in elderly women community-dwelling. Motriz, Rio Claro. 2017; 23(4):1-8. https:// doi.org/10.1590/s1980-6574201700040002

22. Neiva H, Fail L, Izquierdo M, Marques M, Marinho D. The effect of 12 weeks of water-aerobics on health status and physical fitness: An ecological approach. PLoS One. 2018; 13(5): e0198319.

23. Alikhajeh Y, Hosseini S, Moghaddam A. Effects of hydrotherapy in static and dynamic balance among elderly men. Procedia Social and Behavioural Sciences. 2012; 46:2220-4. https://doi. org/10.1016/j.sbspro.2012.05.458

24. Simmons V, Hansen P. Effectiveness of water exercise on postural mobility in the well elderly: An experimental study on balance enhancement. Journal of Gerontology, Medical Sciences. 1996; 51(5):M233-8. PMid: 8808995. https://doi.org/10.1093/ gerona/51A.5.M233

25. Roth A, Miller M, Ricard M, Ritenour D, Chapman B. Comparisons of static and dynamic balance following training in aquatic and land environments. J Sport Rehabil. 2006; 15:299311. https://doi.org/10.1123/jsr.15.4.299

26. Bocalini D, Serra A, Murad N, Levy R. Water-versus landbased exercise effects on physical fitness in older women. Geriatr Gerontol Int. 2008; 8:265-71. PMid: 19149838. https://doi.org/10.1111/j.1447-0594.2008.00485.x

27. Booth C. Water exercise and its effect on balance and gait to reduce the risk of falling in older adults. Activities, Adaptation and Aging. 2004; 28(4):45-57. https://doi.org/10.1300/J016v28n04_04

28. Kaneda K, Sato D, Wakabayashi H, Hanai A, Nomura T. A comparison of the effects of different water exercise programs on balance ability in elderly people. Journal of Aging and Physical Activity. 2008; 16:381-92. PMid: 19033600. https://doi. org/10.1123/japa.16.4.381

29. Sato D, Kaneda K, Wakabayashi H, Nomura T. Comparison of 2 -year effects of once and twice weekly water exercise on activities of daily living ability of community dwelling frail elderly. Archives of Gerontology and Geriatrics. 2009; 49:123-8. PMid: 18804874. https://doi.org/10.1016/j.archger.2008.05.011

30. Abbasi A, Sadeghi H, Tabrizi H, Bagheri K, Ghasemizad A, Asl A. Effect of whole body vibration, aquatic balance and combined training on neuromuscular performance, balance and walking 
ability in male elderly able-bodied individual. World Applied Sciences Journal. 2011; 15(1):84-91.

31. Oh S, Lim J, Kim M, Song W, Yoon B. Comparison of the effects of water- and land-based exercises on the physical function and quality of life in community-dwelling elderly people with history of falling: A single-blind, randomized controlled trial. Archives of Gerontology and Geriatrics. 2015; 60(2):288-93. PMid: 25522928. https://doi.org/10.1016/j.archger.2014.11.001

32. Covill L, Utley C, Hochstein C. Comparison of Ai Chi and impairment-based aquatic therapy for older adults with balance problems: A Clinical study. J Geriatr Phys Ther. 2016; 40(4):204-13.

33. Reichert T, Kanitz A, Delevatti R, Bagatini N, Barroso B, Kruel L. Continuous and interval training programs using deep water running improves functional fitness and blood pressure in the older adults. AGE. 2016; 38(20):1-9. PMid: $26841888 \quad$ PMCid: PMC5005861. https://doi.org/10.1007/s11357-016-9882-5
34. Silva L, Tortelli L, Motta J, Menguer L, Mariano S, Tasca G et al. Effects of aquatic exercise on mental health, functional autonomy and oxidative stress in depressed elderly individuals: A randomized clinical trial. Clinics. 2019; 74(e322):1-7. PMid: 31271585 PMCid: PMC6585867. https://doi.org/10.6061/ clinics/2019/e322

35. Douris P, Southard V, Varga C, Schauss W, Gennaro C, Reiss A. The effect of land and aquatic exercise on balance scores in older adults. Journal of Geriatric Physical Therapy. 2003; 26(1):03-6. https://doi.org/10.1519/00139143-200304000-00001

36. Resende SM, Rassi CM, Viana FP. Effects of hydrotherapy in balance and prevention of falls among elderly women. Rev Bras Fisioter, Sao Carlos. 2008; 12(1):57-63. https://doi.org/10.1590/ S1413-35552008000100011

Table 1. The detailed elaboration of the studies recruited in this systematic review

\begin{tabular}{|c|c|c|c|c|c|c|c|}
\hline $\begin{array}{l}\text { Sr. } \\
\text { No }\end{array}$ & $\begin{array}{l}\text { Author, Year, } \\
\text { Title }\end{array}$ & Journal & Title & Outcome measure & $\begin{array}{l}\text { Period of } \\
\text { intervention }\end{array}$ & Sample size & $p$ value \\
\hline 1 & $\begin{array}{l}\text { Takeshima N } \\
\text { et al } \\
2001 \\
(\mathrm{RCT})^{5}\end{array}$ & $\begin{array}{l}\text { American } \\
\text { College } \\
\text { of Sports } \\
\text { Medicine }\end{array}$ & $\begin{array}{l}\text { Water-based } \\
\text { exercise } \\
\text { improves } \\
\text { health related } \\
\text { aspects of } \\
\text { fitness in older } \\
\text { women }\end{array}$ & $\begin{array}{l}\text { Agility, Flexibility, } \\
\text { Muscular strength for } \\
\text { knee extension, knee } \\
\text { flexion, chest press } \\
\text { and pull, shoulder } \\
\text { press and pull, and } \\
\text { back extension. } \\
\text { Vertical jump, side- } \\
\text { stepping agility, trunk } \\
\text { extension }\end{array}$ & $\begin{array}{l}\text { WEX: 12-wk } \\
\text { supervised WEX } \\
\text { program, } 70 \\
\text { min·day_1, } 3 \text { d.wk_1. }\end{array}$ & $\begin{array}{l}\text { WEX: } 15 \\
\text { Control: } 15\end{array}$ & $\begin{array}{l}\text { Between group } \mathrm{p} \\
\text { value: } \\
\text { Vertical jump } \\
\text { and side stepping } \\
\text { agility test: } \mathrm{p}<0.05\end{array}$ \\
\hline 2 & $\begin{array}{l}\text { Lord S. et al } \\
2006 \\
(\mathrm{RCT})^{6}\end{array}$ & $\begin{array}{l}\text { American } \\
\text { Journal on } \\
\text { Aging }\end{array}$ & $\begin{array}{l}\text { The effects of } \\
\text { water exercise } \\
\text { on physical } \\
\text { functioning } \\
\text { in older people }\end{array}$ & $\begin{array}{l}\text { The maximal } \\
\text { balance range and } \\
\text { coordinated stability } \\
\text { tests, Quadriceps } \\
\text { strength, simple } \\
\text { reaction time and } \\
\text { flexibility }\end{array}$ & $\begin{array}{l}\text { WAVES: } 1 \mathrm{hr} \text { exercise } \\
\text { sessions/week for two } \\
10 \text { week terms }\end{array}$ & $\begin{array}{l}\text { Water exercise } \\
\text { program: } 85+ \\
\text { Control group: } \\
44\end{array}$ & $\begin{array}{l}\text { Maximal } \\
\text { balance } \mathrm{p}<0.01, \\
\text { Coordinated } \\
\text { stability: } \mathrm{p}<0.05, \\
\text { Shoulder } \\
\text { ROM: } \mathrm{p}<0.05, \\
\text { Quadriceps } \\
\text { strength and } \\
\text { reaction time: Not } \\
\text { significant }\end{array}$ \\
\hline 3 & $\begin{array}{l}\text { Tsourlou et } \\
\text { T al } \\
2006 \\
(\mathrm{RCT})^{7}\end{array}$ & $\begin{array}{l}\text { Journal of } \\
\text { Strength and } \\
\text { Conditioning } \\
\text { Research }\end{array}$ & $\begin{array}{l}\text { The effects of } \\
\text { a twenty-four- } \\
\text { week aquatic } \\
\text { training } \\
\text { program on } \\
\text { muscular } \\
\text { strength } \\
\text { performance in } \\
\text { healthy elderly } \\
\text { women }\end{array}$ & $\begin{array}{l}\text { Muscle strength } \\
\text { (isometric and } \\
\text { dynamic) flexibility, } \\
\text { and functional } \\
\text { mobility, Jumping } \\
\text { performance was } \\
\text { evaluated using the } \\
\text { Squat Jump (SJ), } \\
\text { functional mobility } \\
\text { with the timed up- } \\
\text { and-go (TUG I test, } \\
\text { and trunk flexion } \\
\text { with the sit-and-reach } \\
\text { test. }\end{array}$ & $\begin{array}{l}\mathrm{AT}=\text { Supervised } \\
\text { shallow-water } \\
\text { exercise program for } \\
60 \text { minutes/day, } 3 \\
\text { days a week; }\end{array}$ & $\begin{array}{l}\text { Aquatic } \\
\text { training } \\
\text { program: } 12+ \\
\text { Control group: } \\
10\end{array}$ & $\begin{array}{l}\text { TUG and strength } \\
\text { both improved } \\
\text { p }<0.0125\end{array}$ \\
\hline
\end{tabular}




\begin{tabular}{|c|c|c|c|c|c|c|c|}
\hline $\begin{array}{l}\text { Sr. } \\
\text { No }\end{array}$ & $\begin{array}{l}\text { Author, Year, } \\
\text { Title }\end{array}$ & Journal & Title & Outcome measure & $\begin{array}{l}\text { Period of } \\
\text { intervention }\end{array}$ & Sample size & $\mathrm{p}$ value \\
\hline 4 & $\begin{array}{l}\text { Barela A et al } \\
2008 \\
(\mathrm{RCT})^{8}\end{array}$ & $\begin{array}{l}\text { Journal of } \\
\text { Electro- } \\
\text { myography and } \\
\text { Kinesiology }\end{array}$ & $\begin{array}{l}\text { Biomechanical } \\
\text { characteristics } \\
\text { of elderly } \\
\text { individuals } \\
\text { walking } \\
\text { on land and in } \\
\text { water }\end{array}$ & $\begin{array}{l}\text { Spatio-temporal } \\
\text { parameters of gait, } \\
\text { joint angles, ground } \\
\text { reaction force, muscle } \\
\text { activation pattern }\end{array}$ & $\begin{array}{l}10 \text { participants } \\
\text { walked bare feet } \\
\text { at self selected, } \\
\text { comfortable speeds } \\
\text { on both a walkway } \\
\text { on land and in } \\
\text { swimming pool (till } \\
\text { xiphisternum). }\end{array}$ & $\begin{array}{l}\text { In water: } 10+ \\
\text { On land: } 10\end{array}$ & $\begin{array}{l}\text { Elderly group } \\
\text { presented } \\
\text { significantly } \\
\text { shorter stride } \\
\text { duration ( } \mathrm{p}=0.02 \\
\text { between group) } \\
\text { than younger } \\
\text { adults in water. }\end{array}$ \\
\hline 5 & $\begin{array}{l}\text { Candeloro } \\
\text { JM et al } \\
2007 \\
(\mathrm{RCT})^{9}\end{array}$ & $\begin{array}{l}\text { Rev. bras. } \\
\text { fisioter }\end{array}$ & $\begin{array}{l}\text { Effects of a } \\
\text { hydrotherapy } \\
\text { program on } \\
\text { flexibility and } \\
\text { muscular } \\
\text { strength in } \\
\text { elderly women }\end{array}$ & $\begin{array}{l}\text { Flexibility: toe touch, } \\
\text { anterior flexion of } \\
\text { the trunk in seated } \\
\text { position, modified } \\
\text { trunk inclination, } \\
\text { muscle strength } \\
\text { of quadriceps, } \\
\text { hamstring, medial } \\
\text { gluteus and iliopsoas. }\end{array}$ & $\begin{array}{l}\text { The participants were } \\
31 \text { healthy sedentary } \\
\text { elderly women aged } \\
\text { between } 65 \text { and } \\
70 \text { years ( } 16 \text { in the } \\
\text { experimental group } \\
\text { and } 15 \text { in the control } \\
\text { group). }\end{array}$ & $\begin{array}{l}\text { Hydrotherapy } \\
\text { program: } 16+ \\
\text { Control group: } \\
15\end{array}$ & $\begin{array}{l}\mathrm{p} \text { value is not } \\
\text { mentioned. }\end{array}$ \\
\hline 6 & $\begin{array}{l}\text { Melzer I et al } \\
2008 \\
(\mathrm{RCT})^{10}\end{array}$ & BMC Geriatrics & $\begin{array}{l}\text { A water- } \\
\text { based training } \\
\text { program } \\
\text { that include } \\
\text { perturbation } \\
\text { exercises } \\
\text { to improve } \\
\text { stepping } \\
\text { responses in } \\
\text { older adults: } \\
\text { study } \\
\text { protocol for a } \\
\text { randomized } \\
\text { controlled } \\
\text { cross-over trial }\end{array}$ & $\begin{array}{l}\text { Voluntary step } \\
\text { reaction time, } \\
\text { postural stabilogram } \\
\text { diffusion analysis, } \\
\text { Berg Balance Scale, } \\
\text { get up and go test, fall } \\
\text { efficacy scale, Folstein } \\
\text { Mini Mental State } \\
\text { Examination, Late } \\
\text { Life Function and } \\
\text { Disability Index }\end{array}$ & Future aspect study & ------- & $\begin{array}{l}\text { Future aspect } \\
\text { study }\end{array}$ \\
\hline 7 & $\begin{array}{l}\text { Kastura Y } \\
\text { et al } \\
2010 \\
(\mathrm{RCT})^{11}\end{array}$ & $\begin{array}{l}\text { European } \\
\text { Journal of } \\
\text { Applied } \\
\text { Physiology }\end{array}$ & $\begin{array}{l}\text { Effects of } \\
\text { aquatic exercise } \\
\text { training } \\
\text { using water- } \\
\text { resistance } \\
\text { equipment in } \\
\text { elderly }\end{array}$ & $\begin{array}{l}\text { Anthropometric } \\
\text { measurements, } \\
\text { physical performance } \\
\text { test, profile of } \\
\text { mood states, } \\
\text { muscle strength } \\
\text { of plantarflexors, } \\
\text { timed up and go test, } \\
\text { obstacle walking, } \\
\text { walking speed, } \\
\text { walking length. }\end{array}$ & $\begin{array}{l}\text { Aquatic exercise: } \\
\text { using water- } \\
\text { resistance equipment: } \\
\text { The aquatic exercise } \\
\text { training was } 90 \text { min, } \\
\text { three times per week } \\
\text { for } 8 \text { weeks, and } \\
\text { mostly consisted of } \\
\text { walking. }\end{array}$ & $\begin{array}{l}\text { Water resistant } \\
\text { equipment: } \\
12+ \\
\text { Control group: } \\
8\end{array}$ & $\begin{array}{l}\text { Improvements } \\
\text { were observed in } \\
\text { muscle strength } \\
\text { in plantarflexor, } \\
\text { and the timed up } \\
\text { and go test (TUG) } \\
\text { in both groups. } \\
\text { Additionally, } \\
\text { 10-m obstacle } \\
\text { walking and } \\
5 \text {-m maximum } \\
\text { walking speed } \\
\text { and length with } \\
\text { eye-open were } \\
\text { significantly } \\
\text { improved in the } \\
\text { resistance group }\end{array}$ \\
\hline
\end{tabular}




\begin{tabular}{|c|c|c|c|c|c|c|c|}
\hline $\begin{array}{l}\text { Sr. } \\
\text { No }\end{array}$ & $\begin{array}{l}\text { Author, Year, } \\
\text { Title }\end{array}$ & Journal & Title & Outcome measure & $\begin{array}{l}\text { Period of } \\
\text { intervention }\end{array}$ & Sample size & $\mathrm{p}$ value \\
\hline 8 & $\begin{array}{l}\text { Bocalini et al } \\
2010 \\
(\mathrm{RCT})^{12}\end{array}$ & Clinical Science & $\begin{array}{l}\text { Repercussions } \\
\text { of training and } \\
\text { detraining by } \\
\text { water based } \\
\text { exercise on } \\
\text { functional } \\
\text { fitness and } \\
\text { quality of } \\
\text { life: a short- } \\
\text { term follow-up } \\
\text { in healthy older } \\
\text { women }\end{array}$ & $\begin{array}{l}\text { Aerobic power, } \\
\text { neuromuscular fitness } \\
\text { (Arm curl, chair } \\
\text { stand test and 8-foot } \\
\text { up and go test) and } \\
\text { quality of life }\end{array}$ & $\begin{array}{l}\text { Healthy older women } \\
\text { (trained (TR) group) } \\
\text { were submitted to } \\
12 \text { weeks WE (three } \\
45 \text { min sessions per } \\
\text { week) followed by a } \\
\text { 6-week detraining } \\
\text { period. A group } \\
\text { of aged-matched } \\
\text { women without any } \\
\text { exercise training } \\
\text { (UN group) were } \\
\text { evaluated during the } \\
\text { same period }\end{array}$ & $\begin{array}{l}\text { Water exercise: } \\
30+ \\
\text { Control: } 20\end{array}$ & $\begin{array}{l}\text { Balance was } \\
\text { improved at } 4^{\text {th }}, 6^{\text {th }} \\
\text { and even after } 12^{\text {th }} \\
\text { week significantly } \\
\text { with } \mathrm{p}<0.05\end{array}$ \\
\hline 9 & $\begin{array}{l}\text { Avelar N. } \\
\text { et al } \\
2010 \\
(\mathrm{RCT})^{13}\end{array}$ & $\begin{array}{l}\text { Revista } \\
\text { Brasileira de } \\
\text { Fisioterapia }\end{array}$ & $\begin{array}{l}\text { Effectiveness } \\
\text { of aquatic and } \\
\text { non-aquatic } \\
\text { lower } \\
\text { limb muscles } \\
\text { endurance } \\
\text { training in the } \\
\text { static } \\
\text { and dynamic } \\
\text { balance of } \\
\text { elderly people }\end{array}$ & $\begin{array}{l}\text { the Berg Balance } \\
\text { Scale, Dynamic Gait } \\
\text { Index, gait } \\
\text { speed and tandem } \\
\text { gait }\end{array}$ & $\begin{array}{l}36 \text { elderly people } \\
\text { were evaluated } \\
\text { using } 4 \text { tests: the } \\
\text { Berg Balance Scale, } \\
\text { Dynamic Gait } \\
\text { Index, gait speed } \\
\text { and tandem gait. } \\
\text { The participants } \\
\text { were allocated into } \\
3 \text { groups: aquatic } \\
\text { exercise group, non- } \\
\text { aquatic exercise } \\
\text { group and control } \\
\text { group. Aquatic group: } \\
\text { lower-limb muscle } \\
\text { endurance exercise: } \\
\text { 40-minute/day twice } \\
\text { a week for six weeks. }\end{array}$ & $\begin{array}{l}\text { Aquatic group: } \\
14 \\
\text { Non-aquatic } \\
\text { group: } 15 \\
\text { Control group: } \\
17\end{array}$ & $\begin{array}{l}\text { The program for } \\
\text { lower-limb muscle } \\
\text { endurance } \\
\text { significantly } \\
\text { increased balance } \\
\text { (p<0.05) in the } \\
\text { evaluation tests } \\
\text { after the training } \\
\text { program. }\end{array}$ \\
\hline 10 & $\begin{array}{l}\text { Bento P et al } \\
2012 \\
(\mathrm{RCT})^{14}\end{array}$ & $\begin{array}{l}\text { Journal of } \\
\text { Aging and } \\
\text { Physical } \\
\text { Activity }\end{array}$ & $\begin{array}{l}\text { The Effects of a } \\
\text { Water-Based } \\
\text { Exercise } \\
\text { Program on } \\
\text { Strength } \\
\text { and } \\
\text { Functionality } \\
\text { of Older Adults }\end{array}$ & $\begin{array}{l}\text { Peak Torque of } \\
\text { extensor and flexor } \\
\text { of lower extremities } \\
\text { and rate of torque } \\
\text { development of the } \\
\text { ankle, knee and hip } \\
\text { joints. }\end{array}$ & $\begin{array}{l}\text { Thirty-seven elderly } \\
\text { were randomly } \\
\text { assigned to water- } \\
\text { based training ( } 3 \mathrm{~d} / \\
\text { wk for } 12 \mathrm{wk} \text { ) or a } \\
\text { control group }\end{array}$ & $\begin{array}{l}\text { Water based } \\
\text { training: } 24 \\
\text { Control group: } \\
14\end{array}$ & $\begin{array}{l}\text { A peak-torque } \\
\text { increase }(p<.05) \\
\text { was detected for } \\
\text { the hip flexors } \\
\text { and extensors and } \\
\text { for the plantar- } \\
\text { flexor muscles; } \\
\text { The rate of torque } \\
\text { development } \\
\text { increased ( } p<.05) \text {, } \\
\text { Sit and Reach } \\
\text { test, time in } 8 \text {-ft } \\
\text { up-and-go test, } \\
\text { and 6-min test } \\
\text { increased }(p<.05)\end{array}$ \\
\hline
\end{tabular}




\begin{tabular}{|c|c|c|c|c|c|c|c|}
\hline $\begin{array}{l}\text { Sr. } \\
\text { No }\end{array}$ & $\begin{array}{l}\text { Author, Year, } \\
\text { Title }\end{array}$ & Journal & Title & Outcome measure & $\begin{array}{l}\text { Period of } \\
\text { intervention }\end{array}$ & Sample size & $\mathrm{p}$ value \\
\hline 11 & $\begin{array}{l}\text { Elbar O et al } \\
2012 \\
(\mathrm{RCT})^{15}\end{array}$ & $\begin{array}{l}\text { Archives of } \\
\text { Gerontology } \\
\text { and Geriatrics }\end{array}$ & $\begin{array}{l}\text { A water- } \\
\text { based training } \\
\text { program } \\
\text { that includes } \\
\text { perturbation } \\
\text { exercises } \\
\text { improves speed } \\
\text { of voluntary } \\
\text { stepping in } \\
\text { older adults: } \\
\text { A randomized } \\
\text { controlled } \\
\text { cross-over trial }\end{array}$ & $\begin{array}{l}\text { Step execution, } \\
\text { postural stability } \\
\text { parameters in eyes } \\
\text { open and eyes closed } \\
\text { condition }\end{array}$ & $\begin{array}{l}\text { thirty-six } \\
\text { independent old } \\
\text { adults ( } 64-88 \text { years } \\
\text { old) were divided } \\
\text { into two groups. } \\
\text { Group A received } \\
\text { WEP for the first } 12 \\
\text { weeks, followed by } \\
\text { no intervention for } \\
\text { the second } 12 \text { weeks. } \\
\text { Group B did not } \\
\text { receive intervention } \\
\text { for the first } 12 \text { weeks } \\
\text { and received WEP for } \\
\text { the second } 12 \text { weeks. }\end{array}$ & $\begin{array}{l}\text { Initially group } \\
\text { A (18) received } \\
\text { water based } \\
\text { intervention } \\
\text { with } \\
\text { Perturbation } \\
\text { training for } \\
12 \text { weeks and } \\
\text { group B (18) } \\
\text { received no } \\
\text { treatment. } \\
\text { Vice versa was } \\
\text { done for next } \\
12 \text { weeks. }\end{array}$ & $\begin{array}{l}\text { Between group } \\
\text { analysis; foot } \\
\text { contact time } \\
(\mathrm{p}=0.003), \\
\text { step initiation } \\
(\mathrm{p}=0.05) \text { and } \\
\text { swing-phases } \\
(\mathrm{p}=0.002) \text {. An } \\
\text { interaction effect } \\
\text { between group } \\
\text { and time reached } \\
\text { significance for } \\
\text { sway area and } \mathrm{ML} \\
\text { sway range in eyes } \\
\text { open condition } \\
\text { ( } \mathrm{p}=0.04 \text { and } \mathrm{p}= \\
0.05, \text { respectively. } \\
\text { No significant for } \\
\text { between group } \\
\text { and time w.r.t } \\
\text { Berg Balance } \\
\text { scale. }\end{array}$ \\
\hline 12 & $\begin{array}{l}\text { Sanders. M. } \\
2013 \\
(\mathrm{RCT})^{16}\end{array}$ & $\begin{array}{l}\text { Journal of } \\
\text { Sports Science } \\
\text { and Medicine }\end{array}$ & $\begin{array}{l}\text { Impact of the } \\
\text { S.W.E.A.T. } \\
\text { Water-Exercise } \\
\text { Method on } \\
\text { Activities of } \\
\text { Daily Living for } \\
\text { Older Women }\end{array}$ & $\begin{array}{l}\text { Static balance, } \\
\text { dynamic balance, } \\
\text { sit and reach, sit to } \\
\text { stand, walking speed, } \\
\text { agility, stair climb and } \\
\text { biceps curl }\end{array}$ & $\begin{array}{l}\text { Sixty-six women } \\
(60-89 \text { yr of age) self- } \\
\text { selected to a Water } \\
\text { Exercise (WEX) } \\
\text { group ( } \mathrm{n}=48) \text { or } \\
\text { control }(\mathrm{C}) \text { group ( } \mathrm{n} \\
=18) \text {. The training } \\
\text { consisted of a 16- } \\
\text { week ( } 45 \text { min.day-1, } \\
3 \text { d.wk-1) supervised } \\
\text { WEX program that } \\
\text { included } 10 \text { min of } \\
\text { warm-up and warm } \\
\text { down/stretching and } \\
35 \text { min training using } \\
\text { the S.W.E.A.T } \\
\text { method in shallow } \\
\text { water } 1.0-1.2 \text { m, with } \\
\text { water temperature } \\
\text { approximately } 28- \\
29^{\circ} \mathrm{C} \text {. }\end{array}$ & $\begin{array}{l}\text { Water exercise } \\
(48)+ \\
\text { Control group } \\
(18)\end{array}$ & $\begin{array}{l}\text { Sit and reach } \\
(\mathrm{p}<0.05) \text {, Sit to } \\
\text { stand }(\mathrm{p}<0.0001) \text {, } \\
\text { Walk speed } \\
(\mathrm{p}<0.0001) \text {, } \\
\text { walk steps } \\
(\mathrm{p}<0.0001) \text {, agility } \\
(\mathrm{p}<0.000) \text {, arm } \\
\text { curls }(\mathrm{p}<0.0001), \\
\text { static balance } \\
(\mathrm{p}<0.05) \text { and } \\
\text { dynamic balance } \\
(\mathrm{p}<0.0001)\end{array}$ \\
\hline 13 & $\begin{array}{l}\text { Kim S et al } \\
(\mathrm{RCT})^{17}\end{array}$ & $\begin{array}{l}\text { Journal of } \\
\text { Physical } \\
\text { Therapy }\end{array}$ & $\begin{array}{l}\text { Effects of } \\
\text { Aqua Aerobic } \\
\text { Therapy } \\
\text { Exercise for } \\
\text { Older } \\
\text { Adults on } \\
\text { Muscular } \\
\text { Strength, } \\
\text { Agility and } \\
\text { Balance } \\
\text { to Prevent } \\
\text { Falling during } \\
\text { Gait }\end{array}$ & $\begin{array}{l}\text { Muscle strength, } \\
\text { muscle power, } \\
\text { flexibility, agility and } \\
\text { balance. }\end{array}$ & $\begin{array}{l}\text { A total of } 15 \text { subjects } \\
\text { participated in this } \\
\text { study and they were } \\
\text { randomly divided } \\
\text { into the experimental } \\
\text { and the control group }\end{array}$ & $\begin{array}{l}\text { Aqua aerobics: } \\
8 \\
\text { Control group: } \\
7\end{array}$ & $<0.05$ \\
\hline
\end{tabular}




\begin{tabular}{|c|c|c|c|c|c|c|c|}
\hline $\begin{array}{l}\text { Sr. } \\
\text { No }\end{array}$ & $\begin{array}{l}\text { Author, Year, } \\
\text { Title }\end{array}$ & Journal & Title & Outcome measure & $\begin{array}{l}\text { Period of } \\
\text { intervention }\end{array}$ & Sample size & $\mathrm{p}$ value \\
\hline 14 & $\begin{array}{l}\text { Kovách et al } \\
\text { Randomized } \\
\text { clinical trial }^{18}\end{array}$ & $\begin{array}{l}\text { Biomedical } \\
\text { Human } \\
\text { Kinetics }\end{array}$ & $\begin{array}{l}\text { Effects of } \\
\text { Pilates and } \\
\text { aqua fitness } \\
\text { training on } \\
\text { older adults' } \\
\text { physical } \\
\text { functioning } \\
\text { and quality of } \\
\text { life }\end{array}$ & $\begin{array}{l}\text { Fullerton Functional } \\
\text { Fitness Test, } \\
\text { WHO’s Quality of } \\
\text { Life Questionnaire. }\end{array}$ & $\begin{array}{l}\text { A total of } 54 \\
\text { participants }(\mathrm{M}= \\
66.4 \pm 6.2 \text { years }) \text { from } \\
\text { a club for retired } \\
\text { people in Eger, } \\
\text { Hungary. }\end{array}$ & $\begin{array}{l}\text { Aquatic fitness } \\
\text { training: } 17 \\
\text { Pilates: } 22 \\
\text { Control group: } \\
15\end{array}$ & $\begin{array}{l}\text { FITT: } \\
\text { Pilates: strength, } \\
\text { flexibility, } \\
\text { dynamic balance } \\
\text { and aerobic } \\
\text { endurance: } \\
\text { p<0.0001. } \\
\text { Aqua group: body } \\
\text { flexibility: }<0.05 \\
\text { Control } \\
\text { group, body } \\
\text { strength: }<0.01 . \\
\text { WHOQOL-OLD: } \\
\text { Pilates: } \\
\text { Autonomy: }<0.01 \text {, } \\
\text { perception: } \\
<0.001 \\
\text { Aqua group: } \\
\text { sociability, }<0.001\end{array}$ \\
\hline 15 & $\begin{array}{l}\text { Oliveria M } \\
\text { et al } \\
2014 \\
(\mathrm{RCT})^{19}\end{array}$ & $\begin{array}{l}\text { Archives of } \\
\text { Gerontology } \\
\text { and Geriatrics }\end{array}$ & $\begin{array}{l}\text { Effect of } \\
\text { different types } \\
\text { of exercise } \\
\text { on postural } \\
\text { balance in } \\
\text { elderly women: } \\
\text { A randomized } \\
\text { controlled trial }\end{array}$ & $\begin{array}{l}\text { Five postural balance } \\
\text { task were performed } \\
\text { on a force platform } \\
\text { (COP sway), two } \\
\text { legged stand with } \\
\text { eyes open, two legged } \\
\text { stand with eyes } \\
\text { closed, semi-tandem } \\
\text { stand with eyes open, } \\
\text { semi-tendon stand } \\
\text { with eyes close, one } \\
\text { legged stand. }\end{array}$ & $\begin{array}{l}\text { Seventy-four } \\
\text { physically } \\
\text { independent elderly } \\
\text { women, mean age } \\
69+/-4 \text { years, were } \\
\text { randomly assigned } \\
\text { to three intervention } \\
\text { groups. Each group } \\
\text { performed physical } \\
\text { training, including } \\
\text { cardiorespiratory, } \\
\text { muscular strength } \\
\text { and endurance, } \\
\text { flexibility and } \\
\text { sensory-motor } \\
\text { exercises for } 12 \text { weeks }\end{array}$ & $\begin{array}{l}\text { Aquatic } \\
\text { gymnastics: } 28 \\
\text { Mini } \\
\text { trampoline: } 23 \\
\text { Control group: } \\
23\end{array}$ & $\mathrm{p}<0.05$ \\
\hline 16 & $\begin{array}{l}\text { Khanjari Y. } \\
\text { et al } \\
2015 \\
(\mathrm{RCT})^{20}\end{array}$ & $\begin{array}{l}\text { International } \\
\text { Letters of } \\
\text { Social and } \\
\text { Humanistic } \\
\text { Sciences }\end{array}$ & $\begin{array}{l}\text { The effect of } \\
\text { a period of } \\
\text { aquatic therapy } \\
\text { exercise on the } \\
\text { quality of life } \\
\text { and depression } \\
\text { in aged males } \\
\text { suffering } \\
\text { from chronic } \\
\text { physical pains }\end{array}$ & $\begin{array}{l}\text { SF-36 and Depression } \\
\text { questionnaire }\end{array}$ & $\begin{array}{l}30 \text { aged males who } \\
\text { voluntarily referred } \\
\text { to Niyayesh health } \\
\text { center of Shiraz were } \\
\text { selected. They were } \\
\text { randomly divided } \\
\text { in to two groups; } \\
\text { experimental }(\mathrm{n}=15) \\
\text { and control }(\mathrm{n}=15) \text {. } \\
\text { Subjects of aquatic } \\
\text { therapy exercise } \\
\text { were involved in an } \\
8 \text {-week-activity in } \\
\text { water; } 3 \text { sessions per } \\
\text { week each lasting } \\
\text { approximately } 50 \text { to } \\
70 \text { minutes }\end{array}$ & $\begin{array}{l}\text { Aquatic } \\
\text { therapy: } 15 \\
\text { Control group: } \\
15\end{array}$ & $\mathrm{p}<0.05$ in both \\
\hline
\end{tabular}




\begin{tabular}{|c|c|c|c|c|c|c|c|}
\hline $\begin{array}{l}\text { Sr. } \\
\text { No }\end{array}$ & $\begin{array}{l}\text { Author, Year, } \\
\text { Title }\end{array}$ & Journal & Title & Outcome measure & $\begin{array}{l}\text { Period of } \\
\text { intervention }\end{array}$ & Sample size & $p$ value \\
\hline 17 & $\begin{array}{l}\text { Alberti D. } \\
\text { et al } \\
2017 \\
(\mathrm{RCT})^{21}\end{array}$ & $\begin{array}{l}\text { Motriz, Rio } \\
\text { Claro }\end{array}$ & $\begin{array}{l}\text { Water running: } \\
\text { effects on } \\
\text { muscle } \\
\text { function and } \\
\text { functionality }\end{array}$ & $\begin{array}{l}\text { Dynamic isokinetic } \\
\text { strength, lower limb } \\
\text { functionality. }\end{array}$ & $\begin{array}{l}\text { Older women ( } \mathrm{n}= \\
\text { 19) were randomly } \\
\text { assigned to one of } \\
\text { the two groups: } \\
\text { deep-water running } \\
\text { (DWR: } \mathrm{n}=09 \text {, } \\
64.33 \pm 4.24 \mathrm{years,} \\
75.15 \pm 12.53 \mathrm{~kg} \text {, } \\
160.45 \pm 7.52 \mathrm{~cm} \text {; } \\
\text { or control group } \\
\text { CG: } \mathrm{n}=10,64.40 \pm \\
4.22 \text { years, } 74.46 \pm \\
12.39 \mathrm{~kg}, 158.88 \pm \\
5.48 \mathrm{~cm}) . \text { The DWR } \\
\text { group carried out } 18 \\
\text { weeks of deep-water } \\
\text { running, twice } / \text { week } \\
50 \text { min sessions }\end{array}$ & $\begin{array}{l}\text { Deep water } \\
\text { running: } 9 \\
\text { Control group: } \\
10\end{array}$ & $\begin{array}{l}P \text { value not } \\
\text { mentioned }\end{array}$ \\
\hline 18 & $\begin{array}{l}\text { Neiva H. et al } \\
2018 \\
(\mathrm{RCT})^{22}\end{array}$ & $\begin{array}{l}\text { Water-aerobics, } \\
\text { health status } \\
\text { and physical } \\
\text { fitness }\end{array}$ & $\begin{array}{l}\text { The effect of } 12 \\
\text { weeks of water- } \\
\text { aerobics on } \\
\text { health status } \\
\text { and physical } \\
\text { fitness: An } \\
\text { ecological } \\
\text { approach }\end{array}$ & $\begin{array}{l}\text { Strength of upper } \\
\text { limb } \\
\text { Body fat mass } \\
\text { SBP } \\
\text { Triglycerides }\end{array}$ & $\begin{array}{l}\text { Fifteen volunteers } \\
(58.80 \pm 14.32 \text { years } \\
\text { old) were part of an } \\
\text { experimental group } \\
\text { (Exercise), and eight } \\
\text { volunteers ( } 59.00 \pm \\
12.26 \text { years old) were } \\
\text { part of the control } \\
\text { group (Control). The } \\
\text { Exercise performed } \\
45 \text { min of water } \\
\text { aerobics twice a } \\
\text { week for } 12 \text { weeks; } \\
\text { no physical exercise } \\
\text { was permitted for the } \\
\text { Control during the } \\
\text { same period }\end{array}$ & $\begin{array}{l}\text { Water aerobics: } \\
15 \\
\text { Control group: } \\
8\end{array}$ & $\begin{array}{l}\text { The results suggest } \\
\text { that } 12 \text { weeks of } \\
\text { water aerobics } \\
\text { performed twice } \\
\text { a week in a real- } \\
\text { life context seem } \\
\text { to benefit the } \\
\text { explosive strength }\end{array}$ \\
\hline 19 & $\begin{array}{l}\text { Alikhajeh } \\
\text { et al } \\
2012 \\
(\mathrm{RCT})^{23}\end{array}$ & $\begin{array}{l}\text { Procedia } \\
\text { Social and } \\
\text { Behavioural } \\
\text { Sciences }\end{array}$ & $\begin{array}{l}\text { Effects of } \\
\text { hydrotherapy } \\
\text { in static and } \\
\text { dynamic } \\
\text { balance among } \\
\text { elderly men }\end{array}$ & $\begin{array}{l}\text { Sharpened Romberg } \\
\text { test (static balance } \\
\text { with eyes open and } \\
\text { close) and Timed Up } \\
\text { and Go. }\end{array}$ & $\begin{array}{l}\text { The participants were } \\
28 \text { healthy sedentary } \\
\text { elderly men aged } \\
\text { between } 64 \text { and } 79 \\
\text { years }\end{array}$ & $\begin{array}{l}14 \text { in the } \\
\text { experimental } \\
\text { group and } 14 \\
\text { in the control } \\
\text { group }\end{array}$ & $\begin{array}{l}\text { Hydrotherapy } \\
\text { program group: } \\
\text { Sharpened } \\
\text { Romberg test (p } \\
<0.001) \text { and the } \\
\text { Timed Up and Go } \\
\text { test }(\mathrm{p}<0.001) .\end{array}$ \\
\hline 20 & $\begin{array}{l}\text { Simmons V. } \\
\text { et al } \\
1996 \\
\text { Ranomized } \\
\text { clinical trial }^{24}\end{array}$ & $\begin{array}{l}\text { Journal of } \\
\text { Gerontology: } \\
\text { MEDICAL } \\
\text { SCIENCES }\end{array}$ & $\begin{array}{l}\text { Effectiveness of } \\
\text { Water Exercise } \\
\text { on Postural } \\
\text { Mobility } \\
\text { in the Well } \\
\text { Elderly: An } \\
\text { Experimental } \\
\text { Study } \\
\text { on Balance } \\
\text { Enhancement }\end{array}$ & Functional reach & $\begin{array}{l}\text { Four groups of } \\
\text { elderly subjects ( } 80 \\
\pm 5.8 \text { years old) } \\
\text { were placed into } \\
\text { four groups. Each } \\
\text { group met twice } \\
\text { per week for } 45 \\
\text { minutes for } 5 \text { weeks } \\
\text { of simple exercises } \\
\text { or socializing in the } \\
\text { designated medium. }\end{array}$ & $\begin{array}{l}\text { Water } \\
\text { Exercisers: } 13 \\
\text { Land } \\
\text { Exercisers: } 13 \\
\text { Water Sitters: } \\
13 \\
\text { Land Sitters: } \\
13\end{array}$ & $\begin{array}{l}\text { Only water } \\
\text { exercise was } \\
\text { significant over } 5 \\
\text { weeks }\end{array}$ \\
\hline
\end{tabular}




\begin{tabular}{|c|c|c|c|c|c|c|c|}
\hline $\begin{array}{l}\text { Sr. } \\
\text { No }\end{array}$ & $\begin{array}{l}\text { Author, Year, } \\
\text { Title }\end{array}$ & Journal & Title & Outcome measure & $\begin{array}{l}\text { Period of } \\
\text { intervention }\end{array}$ & Sample size & $\mathrm{p}$ value \\
\hline 21 & $\begin{array}{l}\text { Roth A. et al } \\
2006 \\
\text { Randomized } \\
\text { clinical trial }^{25}\end{array}$ & $\begin{array}{l}\text { Journal } \\
\text { of Sports } \\
\text { Rehabilitation }\end{array}$ & $\begin{array}{l}\text { Comparisons } \\
\text { of Static and } \\
\text { Dynamic } \\
\text { Balance } \\
\text { Following } \\
\text { Training in } \\
\text { Aquatic } \\
\text { and Land } \\
\text { Environments }\end{array}$ & $\begin{array}{l}\text { Balance: single leg } \\
\text { stance, } \\
\text { single leg tandem } \\
\text { stance, single leg } \\
\text { stance on medium } \\
\text { density foam and } \\
\text { tandem stance on } \\
\text { medium density } \\
\text { foam. } \\
\text { Centre Of Pressure } \\
\text { angle }\end{array}$ & $\begin{array}{l}24 \text { healthy subjects } \\
\text { randomly } \\
\text { assigned to aquatic } \\
(\mathrm{n}=8) \text {, land }(\mathrm{n}=10) \text {, } \\
\text { or control }(\mathrm{n}=6) \\
\text { groups. } \\
\text { Intervention: Four } \\
\text { weeks of balance } \\
\text { training }\end{array}$ & $\begin{array}{l}\text { Aquatic } \\
\text { exercise: } 8 \\
\text { Land exercise: } \\
10 \\
\text { Control group: } \\
6\end{array}$ & $\begin{array}{l}\text { Results of this } \\
\text { study show that } \\
\text { balance training } \\
\text { can effectively be } \\
\text { performed in both } \\
\text { land and } \\
\text { aquatic } \\
\text { environments. }\end{array}$ \\
\hline 22 & $\begin{array}{l}\text { Bocalini D. } \\
\text { et al } \\
2008 \\
\text { Randomized } \\
\text { clinical trial }^{26}\end{array}$ & $\begin{array}{l}\text { Japan Geriatric } \\
\text { Society }\end{array}$ & $\begin{array}{l}\text { Water- versus } \\
\text { land-based } \\
\text { exercise effects } \\
\text { on physical } \\
\text { fitness in older } \\
\text { women }\end{array}$ & $\begin{array}{l}\text { bodyweight, HR } \\
\text { at rest, maximum } \\
\text { aerobic power and } \\
\text { neuromuscular } \\
\text { performance (upper } \\
\text { and lower body } \\
\text { strength; agility; } \\
\text { upper and lower body } \\
\text { flexibility) }\end{array}$ & $\begin{array}{l}\text { Fifty healthy } \\
\text { sedentary women } \\
\text { were randomly } \\
\text { assigned to sedentary } \\
\text { (S), WE and WL } \\
\text { groups. The two } \\
\text { groups were exercised } \\
\text { for } 12 \text { weeks at } 70 \% \\
\text { of the age-predicted } \\
\text { maximum Heart Rate } \\
\text { (HR). }\end{array}$ & $\begin{array}{l}\text { Water exercise: } \\
27 \\
\text { Walking on } \\
\text { land: } 25\end{array}$ & $\begin{array}{l}\mathrm{VO} 2 \mathrm{max} \text { and } \\
\text { neuromuscular } \\
\text { performance } \\
\mathrm{p}<0.05\end{array}$ \\
\hline 23 & $\begin{array}{l}\text { Booth C. } \\
\text { et al } \\
2008 \\
\text { Randomized } \\
\text { Clinical } \\
\text { Trial }^{27}\end{array}$ & $\begin{array}{l}\text { Activities, } \\
\text { Adaptation and } \\
\text { Aging }\end{array}$ & $\begin{array}{l}\text { Water Exercise } \\
\text { and Its Effect } \\
\text { on Balance and } \\
\text { Gait to Reduce } \\
\text { the Risk of } \\
\text { Falling in } \\
\text { Older Adults }\end{array}$ & $\begin{array}{l}\text { Gait and balance } \\
\text { was assessed with } \\
\text { the Tinetti Gait and } \\
\text { Balance Assessment. } \\
\text { The Health Survey } \\
\text { SF36v2 and a } \\
\text { demographic/health } \\
\text { survey were used to } \\
\text { collect information } \\
\text { concerning overall } \\
\text { health and to identify } \\
\text { other factors } \\
\text { influencing the risk } \\
\text { to fall. }\end{array}$ & $\begin{array}{l}\text { Forty-three women } \\
\text { were recruited, } 20 \\
\text { from land-based and } \\
23 \text { from water-based } \\
\text { exercise programs }\end{array}$ & $\begin{array}{l}\text { Water based } \\
\text { exercise: } 23 \\
\text { Land based } \\
\text { exercise: } 20\end{array}$ & $\begin{array}{l}\text { Water exercise } \\
\text { may contribute a } \\
\text { positive benefit } \\
\text { on balance and } \\
\text { gait for women } 65 \\
\text { years and older }\end{array}$ \\
\hline 24 & $\begin{array}{l}\text { Kaneda K. } \\
\text { et al } \\
2008 \\
\text { Randomized } \\
\text { clinical trial }^{28}\end{array}$ & $\begin{array}{l}\text { Journal of } \\
\text { Aging and } \\
\text { Physical } \\
\text { Activity }\end{array}$ & $\begin{array}{l}\text { A Comparison } \\
\text { of the Effects of } \\
\text { Different } \\
\text { Water Exercise } \\
\text { Programs on } \\
\text { Balance } \\
\text { Ability in } \\
\text { Elderly People }\end{array}$ & $\begin{array}{l}\text { Postural sway test } \\
\text { Tandem walking test } \\
\text { Reaction time } \\
\text { Normal and } \\
\text { maximum walking } \\
\text { test }\end{array}$ & $\begin{array}{l}\text { The participants } \\
\text { completed a twice- } \\
\text { weekly water exercise } \\
\text { intervention for } 12 \\
\text { wk. Exercise sessions } \\
\text { comprised a 10-min } \\
\text { warm-up on land, } 20 \\
\text { min of water-walking } \\
\text { exercise, } 30 \text { min of } \\
\text { water exercise while } \\
\text { separated into NWE } \\
\text { and DWRE, a } 10 \text {-min } \\
\text { rest on land, and } 10 \\
\text { min of recreation and } \\
\text { relaxation in water. }\end{array}$ & $\begin{array}{l}\text { Deep water } \\
\text { running } \\
\text { exercise: } 15 \\
\text { Normal water } \\
\text { exercise: } 15\end{array}$ & $\begin{array}{l}\text { DWRE: Postural } \\
\text { sway distance } \\
(<0.05) \text {, postural } \\
\text { sway area }(<0.05) \text {, } \\
\text { tandem walking } \\
\text { time }(<0.05) \text { and } \\
\text { reaction time } \\
(<0.05) \\
\text { NWE: reaction } \\
\text { time }(<0.05)\end{array}$ \\
\hline
\end{tabular}




\begin{tabular}{|c|c|c|c|c|c|c|c|}
\hline $\begin{array}{l}\text { Sr. } \\
\text { No }\end{array}$ & $\begin{array}{l}\text { Author, Year, } \\
\text { Title }\end{array}$ & Journal & Title & Outcome measure & $\begin{array}{l}\text { Period of } \\
\text { intervention }\end{array}$ & Sample size & $\mathrm{p}$ value \\
\hline 25 & $\begin{array}{l}\text { Sa to D. et al } \\
2009 \\
\text { Randomized } \\
\text { clinical trial }^{29}\end{array}$ & $\begin{array}{l}\text { Archives of } \\
\text { Gerontology } \\
\text { and Geriatrics }\end{array}$ & $\begin{array}{l}\text { Comparison of } \\
2 \text {-year effects of } \\
\text { once and twice } \\
\text { weekly water } \\
\text { exercise } \\
\text { on activities } \\
\text { of daily living } \\
\text { ability of } \\
\text { community } \\
\text { dwelling frail } \\
\text { elderly }\end{array}$ & $\begin{array}{l}\text { ADL ability and lower } \\
\text { muscle strength were } \\
\text { measured before the } \\
\text { beginning of exercise } \\
\text { and } 6 \text { months, } 1 \text { year, } \\
\text { and } 2 \text { years after the } \\
\text { program had started. }\end{array}$ & $\begin{array}{l}\text { Group } 1 \text { participated } \\
\text { in a } 60 \text {-min exercise } \\
\text { session once a week, } \\
\text { for } 2 \text { years, while } \\
\text { Group } 2 \text { attended the } \\
\text { session twice a week. } \\
\text { Exercise sessions } \\
\text { were divided into a } \\
10 \text {-min warm-up on } \\
\text { land and } 50 \text { min of } \\
\text { exercise in water. The } \\
50 \text {-min water exercise } \\
\text { program consisted of } \\
20 \text { min walking, } 10 \\
\text { min ADL exercise, } 10 \\
\text { min stretching and } \\
\text { strength exercises, } \\
\text { and } 10 \text { min relaxation } \\
\text { in water. }\end{array}$ & $\begin{array}{l}\text { Once a week } \\
\text { session: } 10 \\
\text { Twice a week } \\
\text { session: } 12\end{array}$ & $\begin{array}{l}\text { Significant group } \\
\text { differences } \\
\text { occurred for } \\
\text { bathing transfer } \\
\text { and stair climbing } \\
\text { at the 2-year } \\
\text { measurement. } \\
\text { These results } \\
\text { suggested that at } \\
\text { least twice-weekly } \\
\text { water exercise } \\
\text { was necessary to } \\
\text { maintain the ADL } \\
\text { ability and KEX of } \\
\text { the frail elderly }\end{array}$ \\
\hline 26 & $\begin{array}{l}\text { Abbasi A. } \\
\text { et al } \\
2011 \\
\text { Randomized } \\
\text { Clinical } \\
\text { trial }^{30}\end{array}$ & $\begin{array}{l}\text { World Applied } \\
\text { Sciences } \\
\text { Journal }\end{array}$ & $\begin{array}{l}\text { Effect of } \\
\text { Whole Body } \\
\text { Vibration, } \\
\text { Aquatic } \\
\text { Balance and } \\
\text { Combined } \\
\text { Training on } \\
\text { Neuromuscular } \\
\text { Performance, } \\
\text { Balance and } \\
\text { Walking Ability } \\
\text { in Male Elderly } \\
\text { Able-Bodied } \\
\text { Individual }\end{array}$ & $\begin{array}{l}\text { Neuromuscular } \\
\text { performance, balance } \\
\text { (Timed Up and Go } \\
\text { and 5-Chair stand } \\
\text { tests) and walking } \\
\text { ability }\end{array}$ & $\begin{array}{l}\text { Sixty adult male } \\
\text { subjects voluntarily } \\
\text { participated in } \\
\text { WBVT, aquatic } \\
\text { balance- and } \\
\text { combined training. }\end{array}$ & $\begin{array}{l}\text { Aquatic } \\
\text { balance: } 15 \\
\text { Whole body } \\
\text { vibration } \\
\text { technique: } 15 \\
\text { Combined } \\
\text { training on } \\
\text { neuromuscular } \\
\text { performance: } \\
15 \\
\text { Control: } 15\end{array}$ & $\begin{array}{l}\text { Aquatic balance- } \\
\text { and combined- } \\
\text { training are } \\
\text { more persistent } \\
\text { than WBVT } \\
\text { and reduce the } \\
\text { risk for falling } \\
\text { in male able- } \\
\text { bodied elderly } \\
\text { individuals. }\end{array}$ \\
\hline 27 & $\begin{array}{l}\text { Oh. S.et al } \\
2015 \\
\text { Randomized } \\
\text { clinical trial }^{31}\end{array}$ & $\begin{array}{l}\text { Archives of } \\
\text { Gerontology } \\
\text { and Geriatrics }\end{array}$ & $\begin{array}{l}\text { Comparison } \\
\text { of the effects } \\
\text { of water- and } \\
\text { land-based } \\
\text { exercises on } \\
\text { the physical } \\
\text { function and } \\
\text { quality of life } \\
\text { in community- } \\
\text { dwelling } \\
\text { elderly people } \\
\text { with history } \\
\text { of falling: A } \\
\text { single-blind, } \\
\text { randomized } \\
\text { controlled trial }\end{array}$ & $\begin{array}{l}\text { Physical functions } \\
\text { (muscle strength, } \\
\text { flexibility, and } \\
\text { mobility) } \\
\text { And Quality of life } \\
\text { (SF-36) } \\
\text { Fear of falling } \\
\text { (modified- fall } \\
\text { efficacy scale) }\end{array}$ & $\begin{array}{l}\text { Participants were } \\
\text { randomly assigned } \\
\text { to the water-based } \\
\text { exercise group } \\
(\mathrm{n}=34) \text { or land- } \\
\text { based exercise groups } \\
(\mathrm{n}=32) \text {. }\end{array}$ & $\begin{array}{l}\text { Water based: } \\
34 \\
\text { Land based: } 32\end{array}$ & $\begin{array}{l}\text { physical } \\
\text { functioning: } \\
p<0.001 \text {, role } \\
\text { physical: } \\
p<0.001 \text {, role } \\
\text { emotional: } \\
p=0.002 \text {, bodily } \\
\text { pain: } p<0.001 \text {, } \\
\text { vitality: } p<0.001 \text {, } \\
\text { and mental health: } \\
p<0.001) \text {. There } \\
\text { was a significant } \\
\text { difference in the } \\
M-F E S \text { in both } \\
\text { groups } \\
\text { ( } p=0.040) \text {. }\end{array}$ \\
\hline
\end{tabular}




\begin{tabular}{|c|c|c|c|c|c|c|c|}
\hline $\begin{array}{l}\text { Sr. } \\
\text { No }\end{array}$ & $\begin{array}{l}\text { Author, Year, } \\
\text { Title }\end{array}$ & Journal & Title & Outcome measure & $\begin{array}{l}\text { Period of } \\
\text { intervention }\end{array}$ & Sample size & $\mathrm{p}$ value \\
\hline 28 & $\begin{array}{l}\text { Covill L. et al } \\
2016 \\
\text { Randomized } \\
\text { clinical trial }^{32}\end{array}$ & $\begin{array}{l}\text { Journal of } \\
\text { Geriatric } \\
\text { Physical } \\
\text { Therapy }\end{array}$ & $\begin{array}{l}\text { Comparison } \\
\text { of Ai Chi and } \\
\text { Impairment- } \\
\text { Based Aquatic } \\
\text { Therapy for } \\
\text { Older Adults } \\
\text { With Balance } \\
\text { Problems: A } \\
\text { Clinical Study }\end{array}$ & $\begin{array}{l}\text { Physical balance } \\
\text { measures, which } \\
\text { included the Berg } \\
\text { Balance Scale (BBS) } \\
\text { and Timed Up and } \\
\text { Go (TUG), the } \\
\text { Activities-Specific } \\
\text { Balance Confidence } \\
\text { Scale (ABC) and } \\
\text { Numerical Pain } \\
\text { Rating Scale (NPRS), }\end{array}$ & $\begin{array}{l}\text { Thirty-two } \\
\text { community-dwelling } \\
\text { adults, } 65 \text { to } 85 \text { years } \\
\text { old, were referred to } 2 \\
\text { different community } \\
\text { pools for APT. } 15 \\
\text { participants received } \\
\text { Ai Chi-based aquatic } \\
\text { interventions and } 17 \\
\text { subjects received an } \\
\text { IBAT program. }\end{array}$ & $\begin{array}{l}\text { Impairment } \\
\text { Based Aquatic } \\
\text { Therapy: } 15 \\
\text { Ai-Chi: } 17\end{array}$ & $\begin{array}{l}\text { The Berg Balance } \\
\text { Scale and Timed } \\
\text { Up and Go test } \\
\text { showed significant } \\
\text { improvement } \\
\text { (BBS, p =.00; } \\
\text { TUG } \mathrm{p}=.03 \text { ) } \\
\text { after Aquatic } \\
\text { Physical Therapy. } \\
\text { The Activities- } \\
\text { Specific Balance } \\
\text { Confidence Scale } \\
\text { and NPRS did } \\
\text { not improve } \\
\text { significantly } \\
\text { (ABC, } \\
\text { p = .27;NPRS, } \\
\text { p = .77). }\end{array}$ \\
\hline 29 & $\begin{array}{l}\text { Reichert T. } \\
\text { et al } \\
2016 \\
\text { (randomized } \\
\text { clinical } \\
\text { trial) }^{33}\end{array}$ & AGE & $\begin{array}{l}\text { Continuous } \\
\text { and interval } \\
\text { training } \\
\text { programs } \\
\text { using deep } \\
\text { water running } \\
\text { improves } \\
\text { functional } \\
\text { fitness and } \\
\text { blood pressure } \\
\text { in the older } \\
\text { adults }\end{array}$ & $\begin{array}{l}\text { SBP, DBP, foot up- } \\
\text { and-go test, Flexibility } \\
\text { of upper and lower } \\
\text { limbs, strength of } \\
\text { upper extremity and } \\
\text { lower extremities and } \\
\text { 6-min walk test. }\end{array}$ & $\begin{array}{l}\text { Thirty-six individuals } \\
\text { were divided into } \\
\text { continuous group } \\
\text { (CONT) and interval } \\
\text { group (INT). Both } \\
\text { groups were trained } \\
\text { for } 28 \text { weeks (twice } \\
\text { weekly). Measures } \\
\text { were performed } \\
\text { before the training } \\
\text { period, after } 12 \text { weeks } \\
\text { and training period. }\end{array}$ & $\begin{array}{l}\text { Intermittent } \\
\text { therapy: } 18 \\
\text { Continuous } \\
\text { therapy: } 18\end{array}$ & $\begin{array}{l}\text { Foot up-and- } \\
\text { go: }<0.0001, \\
\text { Flexibility } \\
\text { of Lower } \\
\text { limbs: } 0.007, \\
\text { strength } \\
\text { factor: }<0.0001 \\
\text { and } 6 \text {-min test: } \\
0.021 \text { were } \\
\text { significant and } \\
\text { by group: alone } \\
\text { flexibility of upper } \\
\text { limb: } 0.042 \text { was } \\
\text { significant. }\end{array}$ \\
\hline 30 & $\begin{array}{l}\text { Silva L. et al } \\
2017 \\
\text { (randomizer } \\
\text { clinical } \\
\text { trial) }^{34}\end{array}$ & Clinics & $\begin{array}{l}\text { Effects of } \\
\text { aquatic } \\
\text { exercise on } \\
\text { mental health, } \\
\text { functional } \\
\text { autonomy and } \\
\text { oxidative stress } \\
\text { in depressed } \\
\text { elderly } \\
\text { individuals: A } \\
\text { randomized } \\
\text { clinical trial }\end{array}$ & $\begin{array}{l}\text { Depression, anxiety, } \\
\text { Timed up and Go } \\
\text { test, Berg Balance } \\
\text { scale, Flexibility, } \\
\text { and blood based } \\
\text { parameters }\end{array}$ & $\begin{array}{l}92 \text { elderly individuals } \\
\text { were included in } \\
\text { the study and were } \\
\text { allocated into the } \\
\text { depression group } \\
\text { ( } \mathrm{n}=16 \text { ) and non- } \\
\text { depression group } \\
(\mathrm{n}=14) \text {. Both } \\
\text { groups engaged in } \\
\text { the aquatic exercise } \\
\text { program for } 12 \\
\text { weeks, including two } \\
\text { weekly sessions ( } 45 \\
\text { min/session) at a low } \\
\text { intensity (between } \\
50 \% \text { and } 60 \% \text { of } \\
\text { maximal heart rate or } \\
\text { Borg scale scores of } \\
13 \text { to } 14 \text { ) throughout } \\
\text { the intervention. }\end{array}$ & $\begin{array}{l}\text { Based on } \\
\text { depression } \\
\text { subjects were } \\
\text { divided into } \\
\text { two groups: } \\
\text { Depression } \\
\text { group: } 16 \\
\text { Non- } \\
\text { depression } \\
\text { group: } 14\end{array}$ & $\begin{array}{l}\text { TUG: } 0.05 \text {, } \\
\text { BBS: } 0.05 \text { and } \\
\text { Flexibility: } 0.03\end{array}$ \\
\hline
\end{tabular}




\begin{tabular}{|c|c|c|c|c|c|c|c|}
\hline $\begin{array}{l}\text { Sr. } \\
\text { No }\end{array}$ & $\begin{array}{l}\text { Author, Year, } \\
\text { Title }\end{array}$ & Journal & Title & Outcome measure & $\begin{array}{l}\text { Period of } \\
\text { intervention }\end{array}$ & Sample size & p value \\
\hline 31 & $\begin{array}{l}\text { Douris P } \\
\text { et al } \\
--- \\
\text { Randomized } \\
\text { clinical trial }^{35}\end{array}$ & $\begin{array}{l}\text { Journal of } \\
\text { Geriatric } \\
\text { Physical } \\
\text { Therapy }\end{array}$ & $\begin{array}{l}\text { The Effect } \\
\text { of Land and } \\
\text { Aquatic } \\
\text { Exercise on } \\
\text { Balance Scores } \\
\text { in } \\
\text { Older Adults }\end{array}$ & Berg Balance Scale & $\begin{array}{l}11 \text { subjects completed } \\
\text { this study. Five } \\
\text { subjects were from an } \\
\text { assisted living facility } \\
\text { (age } 83.2 \pm 8.1 \text { years) } \\
\text { and } 6 \text { subjects were } \\
\text { from an outpatient } \\
\text { facility (age } 75.0 \pm 3.6 \\
\text { years). Each group } \\
\text { did a comparable } \\
\text { set of lower body } \\
\text { exercises ( } 2 \text { times per } \\
\text { week for } 6 \text { weeks), } \\
\text { but one group } \\
\text { exercised in the } \\
\text { pool, and the other } \\
\text { exercised on land }\end{array}$ & $\begin{array}{l}\text { Aquatic } \\
\text { therapy: } 6 \\
\text { Land therapy: } \\
6\end{array}$ & $\begin{array}{l}\text { There was a } \\
\text { significant main } \\
\text { effect of time (p } \\
<.001) \text { but not } \\
\text { group on BBS } \\
\text { scores }\end{array}$ \\
\hline 32 & $\begin{array}{l}\text { Resende SM. } \\
\text { et al } \\
2008 \\
\text { Experimental } \\
\text { trial }^{36}\end{array}$ & $\begin{array}{l}\text { Rev Bras } \\
\text { Fisioter }\end{array}$ & $\begin{array}{l}\text { Effects of } \\
\text { Hydrotherapy } \\
\text { in balance and } \\
\text { prevention of } \\
\text { falls among } \\
\text { elderly women }\end{array}$ & $\begin{array}{l}\text { Berg Balance Scale } \\
\text { and Timed-up and } \\
\text { Go test }\end{array}$ & $\begin{array}{l}25 \text { elderly women } \\
\text { were evaluated } \\
\text { using two scales: } \\
\text { the Berg Balance } \\
\text { Scale and Timed } \\
\text { Up and Go. The } \\
\text { subjects underwent, } \\
\text { subsequently, a low } \\
\text { to moderate intensity } \\
\text { hydrotherapy } \\
\text { program for balance, } \\
\text { which consisted of } \\
\text { three phases: a phase } \\
\text { of adaptation to the } \\
\text { aquatic environment, } \\
\text { a stretching phase } \\
\text { and a phase of } \\
\text { static and dynamic } \\
\text { balance exercises. } \\
\text { The program was } \\
\text { applied for } 12 \text { weeks, } \\
\text { with two sessions per } \\
\text { week, each session } \\
\text { lasting } 40 \text { minutes. }\end{array}$ & 25 & $\begin{array}{l}\text { Berg Balance } \\
\text { Scale }(\mathrm{p}<0.001) \\
\text { and the Timed Up } \\
\text { and Go test }(\mathrm{p}< \\
0.001) .\end{array}$ \\
\hline
\end{tabular}

